\title{
Choroidal osteoma (osseous choristoma): an atypical
} case

G Cennamo, G Iaccarino, G de Crecchio, G Liguori

Università degli Studi di Napoli, I Facultà di

Medicina e Chirurgia, Istituto di Clinica

Oculistica

G Iaccarino

II Facultà di Medicina e Chirurgia

Istituto di Oftalmologia G Cennamo

$\mathrm{G}$ de Crecchio

G Liguori

Correspondence to:

Professor G Cennamo, Clinica Oculistica, II Policlinico, via Pansini 5, Napoli, Italy.

Accepted for publication 22 March 1990

Figure 2 B-scan echogram showing a solid mass lesion of extremely high reflectivity shadowing all the structures behind it. It is usually diagnosed during the second and third decades of life. ${ }^{2}$

Ophthalmoscopy shows a circumscribed, irregularly ovoid or round mass with defined borders. The colour is related to the degree of thinning and depigmentation of the overlying retinal pigment epithelium, ${ }^{3}$ varying from a diffuse reddish orange, thus resembling choroidal haemangioma, to a yellowish white coloration which gradually shades peripherally into pinkish yellow, becoming indistinguishable from the surrounding healthy area. ${ }^{4}$

We report here a case of choroidal osteoma which, because of its atypical features, presented particular diagnostic problems.

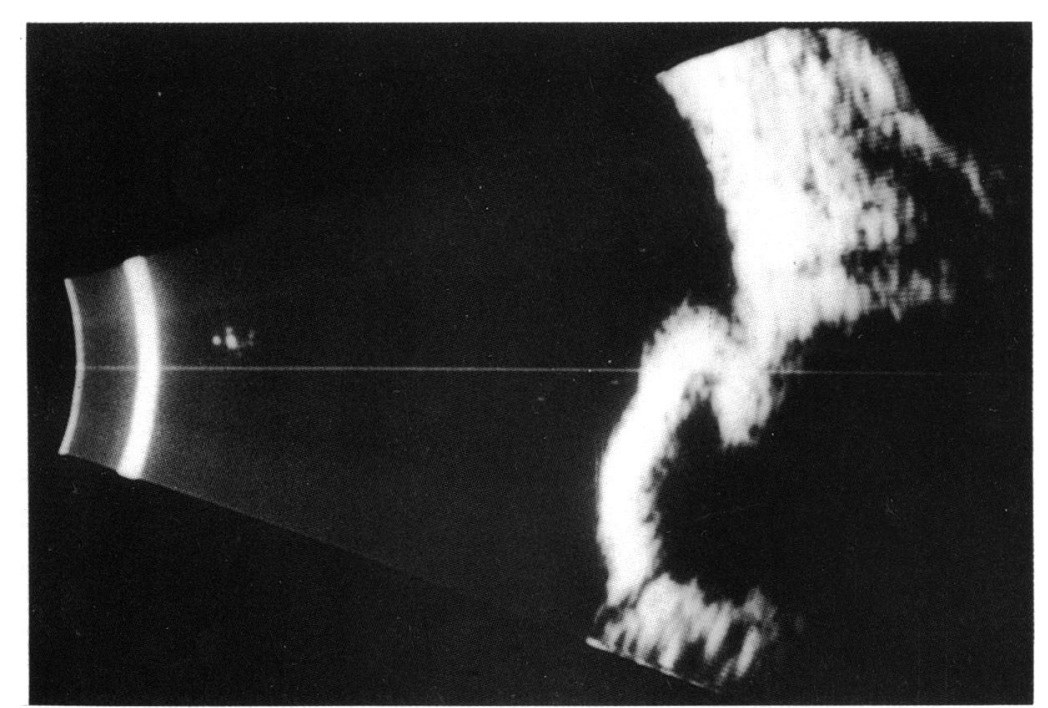

\begin{abstract}
A case of choroidal osteoma presenting in a 22-year-old girl is reported. The tumour, unilateral and in a juxtapapillary site, appeared markedly elevated on the retinal plane, not flat or slightly elevated as in previous reports. Visual acuity was not affected, and there was a complete absence of subjective symptoms. Echography, fluorangiography, computerised tomography, and visual field tests were performed. Echography is the best method for identifying and differentiating this lesion from a malignant tumour.
\end{abstract}

Choroidal osteoma, or osseous choristoma, was first described by Gass et al in 1978.' It is usually present from birth and typically has a low development potential. It presents unilaterally in most cases, has a predilection for the female sex, and favours a juxtapapillary location, becoming clinically manifest when it involves the macula.

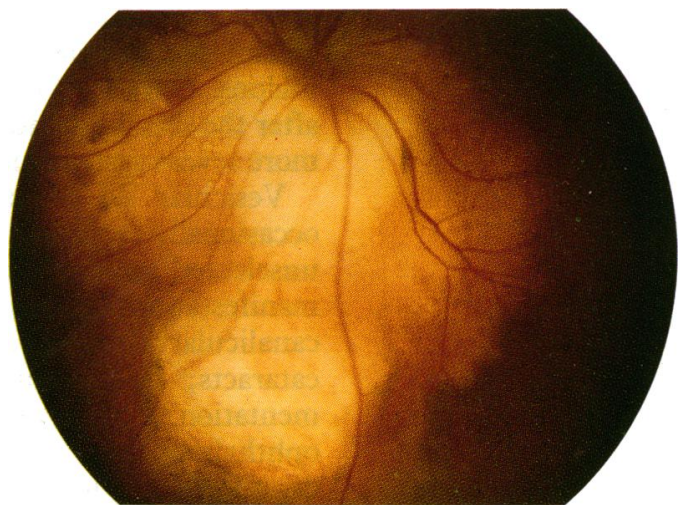

Figure I Left eye. A solid mass located in the juxtapapillary region of the lower nasal quadrant. The mass had a yellowish white centre and was elevated from the retinal plane.

\section{Case report}

A 22 year-old-girl presented without any previous relevant medical history. The right eye was normal. In the left eye the visual acuity was $60 / 60$ and the anterior segment normal. A fundus examination revealed a solid mass of approximately $6 \times 6$ papillary diameters located in the juxtapapillary region of the lower nasal quadrant. The mass was elevated from the retinal plane by about 5 dioptres and had a yellowish white centre and pinkish yellow sloping edges (Fig 1). Standardised echography ${ }^{5}$ showed the lesion as a solid, raised subretinal mass with an extremely high reflectivity, observable even at the anterior surface of the lesion and shadowing the sclera and also the posterior orbital tissues ${ }^{6}$ (Fig 2). The echographic images of the lesion were of foreign body type, showing spikes of extremely high reflectivity from the retinal surface corresponding to the tumour site (Fig 3). In the remaining areas the thickness of the retina and choroid was normal; the optic nerve was also of normal diameter and reflectivity.

Fluorescein angiography showed a pattern of irregular hyperfluorescence overlying the tumour and evidence of diffuse staining of the tumour surface during the later stages. Numerous capillaries on the surface of the choroidal osteoma were present in the early phase of the angiogram (Fig 4). CT showed a radiodense lesion adjacent to the left optic nerve head, at the posterior pole, of the same density as normal bone (Fig 5).

When the patient was seen a year after the first observation, no variations of the mass were noted.

\section{Discussion}

The 5 dioptres elevation from the plane renders this case atypical. The clinical picture of this tumour - its location, unilaterality, its arising from a single focus, and the patient's age when it 


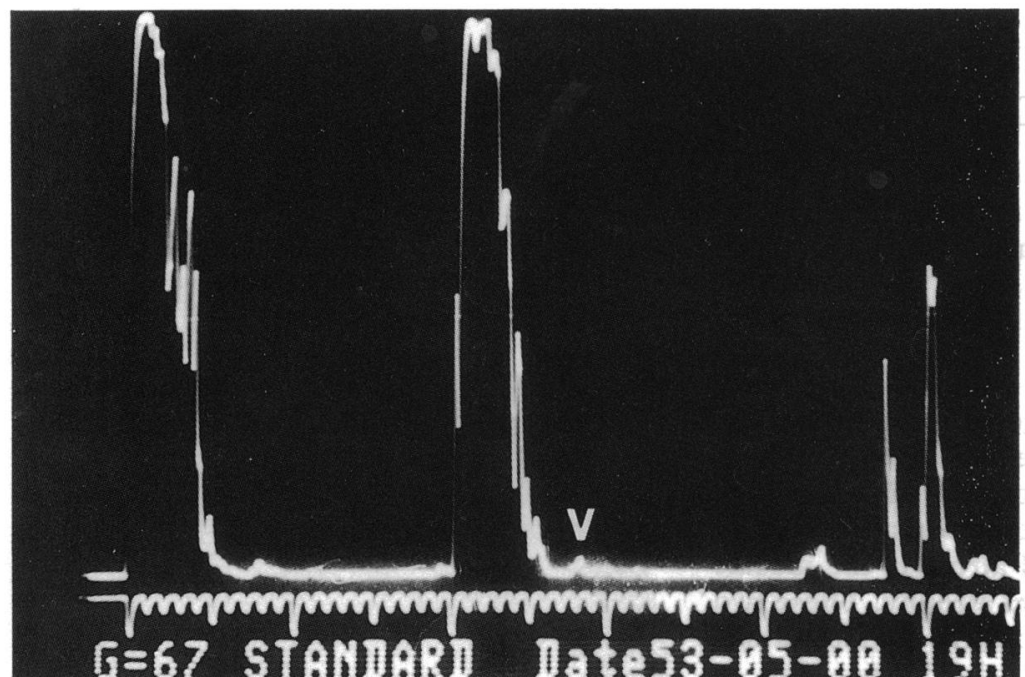

Figure 3 Echogram obtained with standardised A-scan echography showing a lesion of very high reflectivity and posterior shadowing due to the presence of bone tissue in the choroidal layer. The arrow indicates a very low spike obtained from the outer sclera.

was first observed facilitated its diagnostic differentiation from metastatic carcinoma, poorly pigmented malignant choroidal melanoma, optic nerve tumours involving the posterior pole of the eye, choroid ossification (typical of the phthisical eye), retinoblastoma, and choroid haemangioma. But it is echography that allows an exact differential diagnosis to be made. Ultrasonically osteoma presents as a solid mass with a single spike of extremely high reflectivity which is not spontaneously mobile, with marked acoustic shadowing of the tissue behind the lesion.

Ultrasonically malignant melanoma presents with a regular, medium to low reflectivity with sudden rapid, spontaneous movements of the spikes indicating the presence of abnormal vascularisation. In contrast, the reflectivity of the metastatic carcinoma is medium to high and irregular, and spontaneous movements of the spikes are absent. With both these lesions, when shadowing of the posterior orbital tissues is

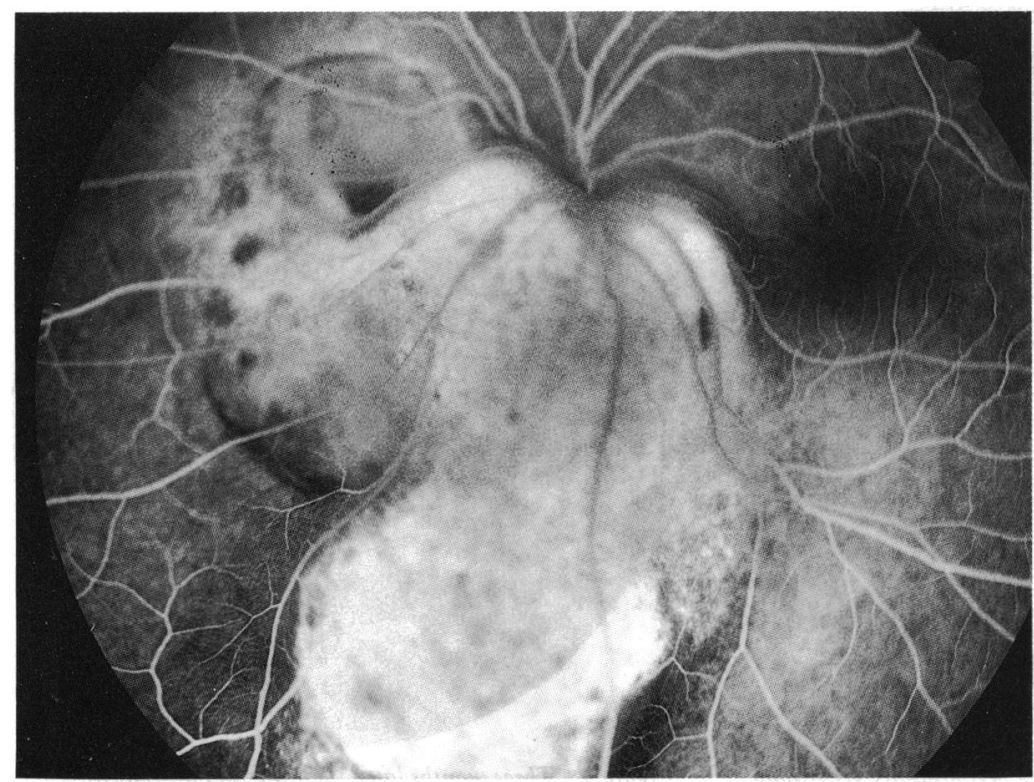

Figure 4 Fluorescein angiography showing a pattern of irregular hyperfluorescence overlying the tumour in the venous phase.

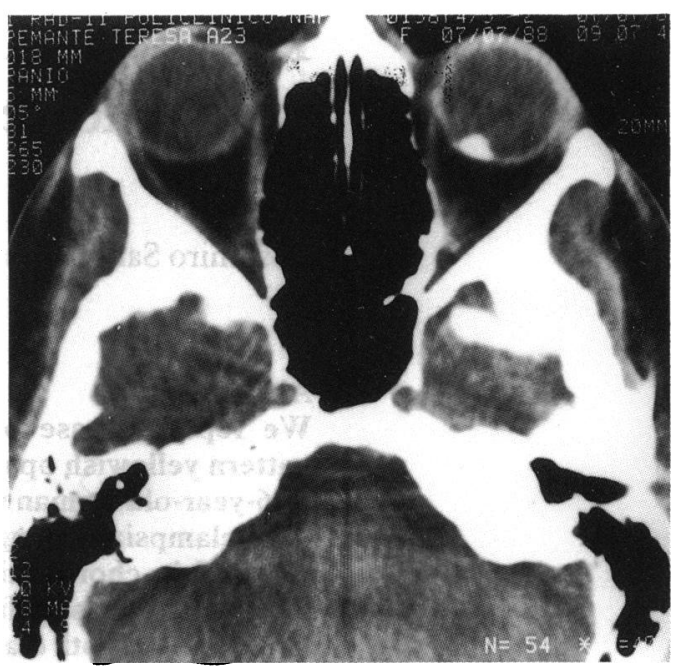

Figure 5 Computerised axial tomography scan showing the presence of a bony plaque in the juxtapapillary region adjacent to the left optic nerve head.

present it is never so pronounced as it is in osteoma. The presence of a normal optic nerve with standardised A scan rules out the presence of a primitive neoplastic tumour of this structure.

Ossification of the choroid is typical of the phthisical eye. The phthisical eye, because of increased thickness and folds of the choroid, shows a shadowing of the posterior orbital tissues. Moreover this shadowing may be due to calcification of the choroid, which is present in a very advanced stage of the disease and distributed in a mottled fashion, not in a single mass as in osteoma. In retinoblastoma, in contrast to choroid osteoma, there is no single spike of high reflectivity of foreign body type but rather a series of spikes of high reflectivity originating both from the surface and from the neoplastic mass. These are referable to the presence of irregularly distributed calcification inside the tumour. ${ }^{8}$

In conclusion, owing to clinical similarities with osteoma serious problems of differential diagnosis are encountered with calcified haemangioma of the choroid. CT and echography makes identification and differentiation of osteoma and calcified haemangioma possible. Osteoma presents as a single, compact, calcified mass, while calcified haemangioma presents as bone surrounded by many large vascular channels. $^{9}$

1. Gass JDM, Guerry RK, Jack RL, Harris C. Choroidal osteomas. Arch Ophthalmol 1978; 96: 428-35.

2 Baum M, Pinkerton AR, Berler DK, Kramer KK. Choroidal osteomas. Ann Ophthalmol 1979; 11: 1849-51.

$\mathrm{Mc}$ Leod BK. Choroidal osteomas presenting in pregnancy. $\mathrm{Br}$ f Ophthalmol 1988; 72: 612-4.

4 Gass JDM New observations concerning choroidal osteomas. Int Ophthalmol 1979; 1: 71-84.

5 Ossoinig KC. In: Dallow CRL, ed. Standardised echography: basic principles, clinical applications and results in ophthalmic ultrasonography: comparative techniques Int Ophthalmol Clin 1979; 19: 127.

6 Santoni G, Ricci AL, Lupidi G, Ciorba E, Fiore C. Choristome osseux de la choroide bilateral et microphtalmie asymetrique. f Fr Ophtalmol 1988; 11: 363-7.

7 Ossoinig KC, Cennamo G, Frazier-Byrne S. Echographic differential diagnosis of optic nerve lesion. Doc Ophthalmol Proc Ser 1981; 29: 327-32.

8 Ossoinig KC, Cennamo G, Green RL, Weyer SML. Echographic results in the diagnosis of retinoblastoma. Doc Ophthalmol Proc Ser 1981; 29: 103-7.

9 Reese AB. Tumours of the eye. 3rd ed. New York: Harper and Row, 1976: 266-7. 